\title{
Use of the Hamilton Rating Scale for Depression and the Carroll Self-Rating Scale in Adolescents
}

\author{
Douglas R. Robbins, Norman E. Alessi, Marit V. Colfer, and Gordon W. \\ Yanchyshyn
}

Received April 20, 1984; revised version received July 16, 1984; accepted August 5, 1984.

\begin{abstract}
The relationship of clinicians' ratings of depression in adolescents to self-rating is important to clinical research. The Hamilton Rating Scale for Depression (HRSD) and the Carroll Self-Rating Scale (CSRS) were compared in a study of 81 psychiatrically hospitalized adolescents. The correlation of CSRS and HRSD total scores in adolescents with melancholic major depression was 0.46 , lower than the correlation reported in adults $(0.80)$. Higher correlations were seen in females and in nonmelancholic and nondepressed subgroups. While the comparison of the two rating methods suggests some characteristics of depressed adolescents' presentation of their illness, it does not appear that the self-rating instrument can be used as an alternative to clinicians' ratings.
\end{abstract}

Key Words. Adolescents, depression, rating scales, Hamilton Rating Scale for Depression.

The Carroll Self-Rating Scale (CSRS) was developed as a self-rating instrument to parallel the widely used Hamilton Rating Scale for Depression (HRSD) (Hamilton, 1960). The CSRS assesses the same 17 symptoms as the HRSD, and both scales have a maximum score of 52. The development and validation of the CSRS have been described in depth (Carroll et al., 1981; Feinberg et al., 1981; Smouse et al., 1981).

Depression has now been established as a significant source of psychiatric illness in adolescence (Hudgens, 1974; Strober et al., 1981; Robbins et al., 1982). The common difficulties in diagnosis and assessment of affect in adolescents make it particularly important that experience with rating scales be understood. An examination of differences between adolescents and adults in their responses to rating scales may also shed light on possible age-related differences in the presentation of depression.

Carroll et al. (1981) found a correlation of HRSD and CSRS total scores of 0.80 $(p<0.001)$ in a sample of inpatients with endogenous depression. Correlations of specific symptoms on the HRSD and CSRS were variable, ranging from -0.06 (loss of insight, denial) and 0.23 (psychomotor agitation) to 0.67 (depressed mood), 0.72 (suicidality), and 0.73 (initial insomnia). Carroll et al. (1981) interpreted their results as suggesting that the CSRS reflected a subjective dimension not assessed by the

\footnotetext{
Douglas R. Robbins, M.D., and Norman E. Alessi, M.D., are Assistant Professors, and at the time of the study, Marit V. Colfer, M.D., was Instructor and Gordon W. Yanchyshyn, M.D., was a Fellow in Child Psychiatry, Department of Psychiatry, University of Michigan. (Reprint requests to Dr. D.R. Robbins, Adolescent Psychiatry Service, University of Michigan Hospital, Ann Arbor, MI 48109, USA.)
} 
HRSD and that the two scales were complementary. The CSRS was proposed as a "credible alternative" to the HRSD in the assessment of severity of depression (Smouse et al., 1981). Patients with nonendogenous depression were found to have higher self-ratings (CSRS) than clinicians' rating (HRSD), with correspondingly lower correlations of total scores (0.66). The endogenous depressives' CSRS scores increased more rapidly than their HRSD scores with increasing severity of depression, leading to lower correlations in more severe depression (Feinberg et al., 1981).

There have been few other comparisons of clinicians' ratings and self-ratings of depression. Bailey and Coppen (1976) found that there was a satisfactory correlation between the HRSD and the Beck Depression Inventory in two-thirds of adult depressives, but results were quite discrepant in the other third. In a study of adult depressed patients during the acute episode and 10 months later when most were recovered, Prusoff et al. (1972) administered the HRSD and a 110-item self-report inventory, part of which was a modification of the Symptom Checklist (SCL) (Raskin et al., 1967). They observed that concordance between clinicians' assessments and self-reports was low during the acute episode ( 0.31 for depressed mood) but improved at followup (0.67). No such studies have been reported in depressed adolescents.

The present article presents the correlations between HRSD and CSRS total scores and scores for specific symptoms in an inpatient adolescent psychiatry population.

\section{Methods}

Eighty-one adolescent psychiatric inpatients (ages 13-18) in the University of Michigan Adolescent Psychiatry Service were assessed with the HRSD by two child psychiatrists based on a structured interview within the first 2 weeks of admission. The CSRS was given immediately after the interview.

Research Diagnostic Criteria (RDC) (Spitzer et al., 1978) and DSM-III (American Psychiatric Association, 1980) diagnoses were assigned by consensus of the two child psychiatrists, based on the interview and all other clinical data. The application of the RDC to this adolescent population has been described elsewhere (Robbins et al., 1982).

\section{Results}

Interrater reliability on the HRSD was 0.90 . Total HRSD and CSRS scores for the 81 patients, all with major depressive disorders (MDD), melancholic (MDD-Mel), nonmelancholic (MDD/Non-Mel), nondepressed (Non-Dep), male and female patients, are presented in Table 1, and values are displayed in Fig. 1. Mean CSRS totals are higher than HRSD totals in all groups except the MDD-Mel group. The large standard deviations of HRSD totals, including the MDD groups, reflect the fact that not all patients with MDD were currently in the most severe phase of a depressive episode. The correlation between HRSD and CSRS totals for all patients was 0.56 . For the MDD-Mel groups, the correlation was 0.46 , as compared with a correlation of 0.80 reported by Carroll et al. (1981) for adults. Correlations were higher for the MDD/Non-Mel (0.59) and Non-Dep groups (0.76). Males had a somewhat higher correlation than females, but both attained the same level of statistical significance. 
Table 1. Hamilton Rating Scale for Depression and Carroll Self-Rating Scale total scores

\begin{tabular}{|c|c|c|c|c|c|c|c|}
\hline \multirow[b]{2}{*}{ Diagnosis } & \multirow[b]{2}{*}{$n$} & \multicolumn{2}{|c|}{ Hamilton 1} & \multicolumn{2}{|c|}{ Carroll } & \multirow[b]{2}{*}{ Correlation } & \multirow{2}{*}{$\begin{array}{c}\text { Significance } \\
(p)\end{array}$} \\
\hline & & Mean & so & Mean & SD & & \\
\hline All patients & 81 & 14.15 & 7.57 & 16.96 & 9.79 & 0.56 & 0.00001 \\
\hline All major depressive disorder & 31 & 18.16 & 8.30 & 20.45 & 10.36 & 0.42 & 0.02 \\
\hline $\begin{array}{l}\text { Major depressive disorder, } \\
\text { melancholic }\end{array}$ & 20 & 19.90 & 8.22 & 19.10 & 9.37 & 0.46 & 0.04 \\
\hline $\begin{array}{l}\text { Major depressive disorder, } \\
\text { nonmelancholic }\end{array}$ & 11 & 15.00 & 7.85 & 22.91 & 12.04 & 0.59 & 0.06 \\
\hline Nondepressed 2 & 25 & 9.76 & 4.92 & 16.04 & 8.68 & 0.76 & 0.00001 \\
\hline Female & 47 & 15.06 & 8.64 & 17.81 & 10.74 & 0.53 & 0.0001 \\
\hline Male & 34 & 12.88 & 5.68 & 15.79 & 8.31 & 0.64 & 0.0001 \\
\hline
\end{tabular}

1. Interrater reliability on Hamilton Rating Scale for Depression $=0.90$ (Pearson correlation coefficient). 2. Nondepressed category excludes all patients with primary diagnosis of major depressive disorder, bipolar affective disorder, schizoaffective disorder, dysthymic disorder, or cyclothymic disorder.

Correlations of HRSD and CSRS ratings of specific symptoms are presented in Table 2, along with the corresponding correlation coefficients for melancholic adults. In the MDD-Mel groups, only the correlations for the symptoms of guilt, initial, middle, and delayed insomnia, somatic anxiety, and appetite attained statistical significance, and all symptoms had lower correlations than those found in the adult sample of Carroll et al. (1981) except somatic anxiety, which was higher $(0.55$ in

Fig. 1. Carroll Self-Rating Scale (CSRS) and Hamilton Rating Scale for Depression (HRSD) total scores

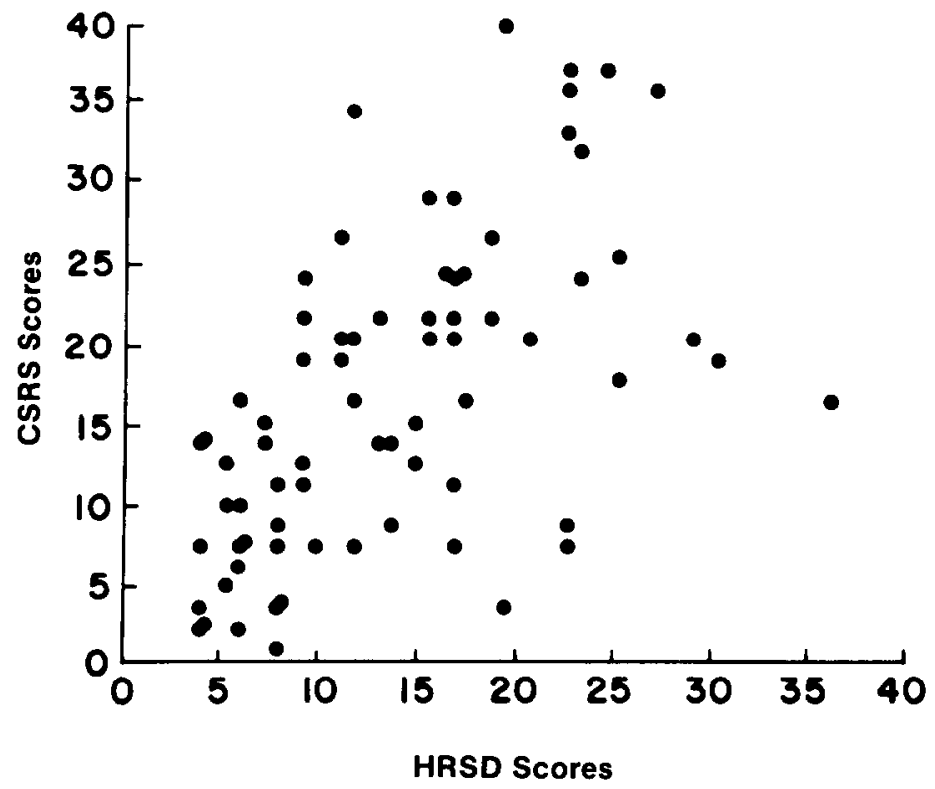




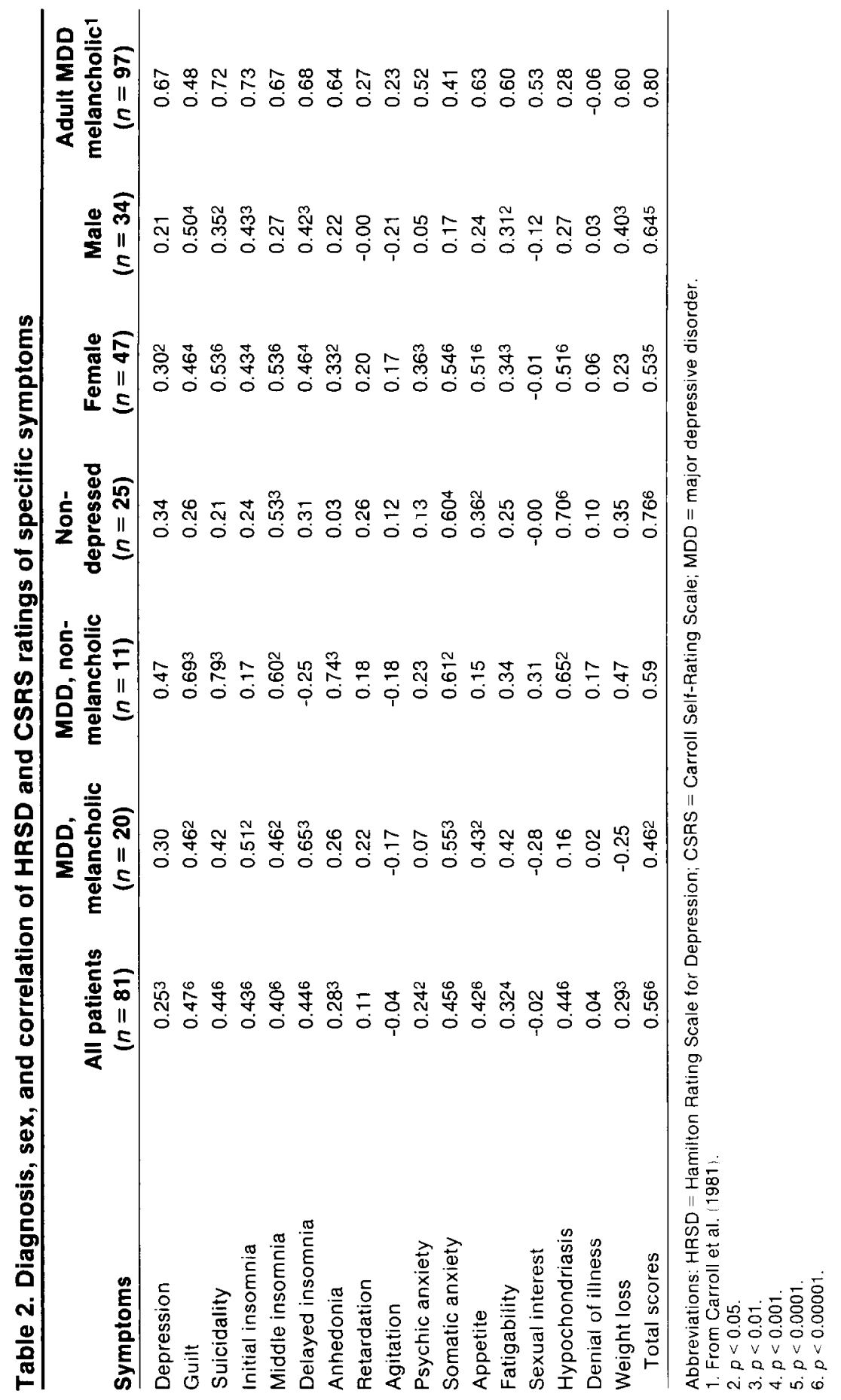


adolescents vs. 0.41 in adults). For some key symptoms, the difference in correlations between adults and adolescents was quite striking, e.g., depression ( 0.30 in adolescents vs. 0.67 in adults) and anhedonia ( 0.26 in adolescents vs. 0.64 in adults). Girls showed significant correlations between the two scales on many more symptoms than did boys, although the correlations of total scores did not reflect this.

The correlation ratings for all HDRS and CSRS symptoms in the MDD-Mel group were examined and are available from the authors upon request. Self-reports for only initial insomnia, delayed insomnia, and somatic anxiety correlated better than 0.5 with the total HRSD. Interestingly, HRSD ratings of depressed mood correlated better than 0.5 only with CSRS scores related to work and interest (anhedonia) and to denial.

One possible explanation for the low correlation between the two rating scales is low variance on the individual items in the scales. Table 3 presents the mean values and standard deviations for all items on both the HRSD and the CSRS, for all patients. The relatively large standard deviations for the scales reflect a high variance on most of the individual items. Thus, low item variance does not appear to be a factor in the overall correlations.

Table 3. Mean values and standard deviations for HRSD and CSRS ratings of specific symptoms, for all patients $(n=81)$

\begin{tabular}{lccccc}
\hline & \multicolumn{2}{c}{ HRSD } & & \multicolumn{2}{c}{ CSRS } \\
\cline { 2 - 3 } \cline { 5 - 6 } Symptoms & Mean & SD & & Mean & SD \\
\hline Depression & 1.75 & 1.13 & & 1.09 & 1.28 \\
Guilt & 1.28 & 1.05 & & 0.98 & 1.22 \\
Suicidality & 1.20 & 1.29 & & 0.63 & 1.19 \\
Initial insomnia & 0.84 & 0.91 & & 0.58 & 0.82 \\
Middle insomnia & 0.43 & 0.74 & & 0.61 & 0.77 \\
Delayed insomnia & 0.30 & 0.66 & & 0.48 & 0.78 \\
Anhedonia & 1.54 & 1.33 & & 1.31 & 1.29 \\
Retardation & 0.91 & 0.96 & & 1.16 & 1.39 \\
Agitation & 0.36 & 0.64 & & 1.27 & 1.19 \\
Psychic anxiety & 1.28 & 1.16 & & 0.98 & 1.14 \\
Somatic anxiety & 1.07 & 1.07 & & 0.99 & 1.19 \\
Appetite & 0.53 & 0.74 & & 0.51 & 0.72 \\
Fatigability & 0.70 & 0.75 & & 0.55 & 0.72 \\
Sexual interest & 0.12 & 0.43 & & 0.55 & 0.85 \\
Hypochondriasis & 0.55 & 0.82 & & 0.72 & 0.98 \\
Denial of illness & 0.60 & 0.70 & & 0.39 & 0.56 \\
Weight loss & 0.65 & 0.87 & & 0.34 & 0.67 \\
$\quad$ Total & 14.15 & 7.57 & & 16.96 & 9.79 \\
\hline
\end{tabular}

Abbreviations: HRSD = Hamilton Rating Scale for Depression; CSRS = Carroll Self-Rating Scale.

\section{Discussion}

In comparison with the concordance between clinicians' ratings (HRSD) and selfreports (CSRS) in the adult endogenous sample of Carroll et al. (1981), adolescents appear to show much lower agreement between the two measures of depression. 
Certainly, it could not be said that the CSRS can be used as an alternative to HRSD ratings in adolescents.

The results may not simply reflect a difference between adolescents and adults, however, for the correlations seen in our sample are similar to those seen by Prusoff et al. (1972) in their adult population during the acute episode. Carroll et al. (1981) did not specify whether their patients were in acute episodes, although this is implied. The fact that Prusoff et al. (1972) used different self-report measures, however, raises questions about the comparability of our results, and the more valid comparison must be considered to be with the study by Carroll et al. (1981). If so, depressed adolescents appear less likely than adults to divulge on a self-report scale symptoms of depression that were evident in a clinicians' interview.

The apparently lower correlation conceivably may result from a number of factors other than age of the subjects. The cognitive capacity to read, understand, and complete the self-rating scale may differ. Demographic factors or the clinical subtypes of depression may vary between the populations studied and may affect the correlation. Styles of interpersonal interactions and readiness or reluctance to respond to a self-report candidly may also partially account for the lower correlations.

The better correlations between the self-ratings and clinicians' ratings on initial and delayed insomnia and somatic anxiety should be noted. These would appear to be symptoms that adolescents are more likely to be conscious of and to report. It is interesting that somatic symptoms, such as headache and recurrent abdominal pain, have long been considered to be one of the "depressive equivalents" or "masks" of depression in children and adolescents (Carlson and Cantwell, 1980). There may be reasons for this observation other than the selective attention of clinicians to certain symptoms. Our findings suggest that some depressed adolescents may be more direct and accurate in their communication of somatic symptoms than they are in reporting depressed mood, anhedonia, or most other depressive symptoms.

The higher correlations shown by girls relative to boys on many symptoms, particularly suicidal feelings, insomnia, somatic anxiety, appetite, and hypochondriasis, may reflect an important difference in how girls experience depression.

It is also notable that adolescents with depressed mood as rated by clinicians did not rate themselves consistently as depressed on the self-rating instrument, but were more likely to rate themselves as anhedonic (e.g., "Lately I don't feel like going out with my friends") and to give answers indicating denial (e.g., "If people got off my back, I would be okay"). It would appear that clinicians should rely less on the adolescent's direct statements regarding mood and inquire more about the past areas of interest and pleasure.

Our observations, then, suggest that the CSRS not be used as an alternative to the HRSD in studies of the severity of depression in adolescents. The CSRS is an important reflection of the depressed adolescent's subjective experience, however, and its inclusion in clinical studies enriches our observations. Serial ratings, for instance, sometimes show one patient's CSRS declining in parallel with his HRSD as he improves, while another patient's CSRS remains high despite improvement on the HSRD. Both patients have had an improvement in their depressive symptoms, but the latter remains subjectively distressed, and it is important that we be aware of the 
distinction. Further studies on the role of personality disorder (cf. Friedman et al., 1982; McManus et al., 1984), as well as more detailed examination of the role of severity of depression, age within the adolescent range, and sex, will help us to understand these issues more clearly.

\section{References}

American Psychiatric Association. DSM-III: Diagnostic and Statistical Manual of Mental Disorders. 3rd ed. APA, Washington, DC (1980).

Bailey, J., and Coppen, A. A comparison between the Hamilton rating scale and the Beck inventory in the reassessment of depression. British Journal of Psychiatry, 128, 486 (1976).

Carlson, G.A., and Cantwell, D.P. Unmasking masked depression in children and adolescents. American Journal of Psychiatry, 137, 445 (1980).

Carroll, B.J., Feinberg, M., Smouse, P.E., Rawson, S.G., and Greden, J.F. The Carroll Rating Scale for Depression: I. Development, reliability and validation. British Journal of Psychiatry, 138, 194 (1981).

Feinberg, M., Carroll, B.J., Smouse, P.E., and Dawson, S.G. The Carroll Rating Scale for Depression: III. Comparison with other rating instruments. British Journal of Psychiatry, 38, 205 (1981).

Friedman, R., Clarkin, J., Corn, R., Aronoff, M., Hurt, S., and Murphy, M. DSM-III and affective pathology in hospitalized adolescents. Journal of Nervous and Mental Disease, 170, 511 (1982).

Hamilton, M. A rating scale for depression. Journal of Neurology, Neurosurgery, and Psychiatry, 23, 56 (1960).

Hudgens, R.W. Psychiatric Disorders in Adolescence. Williams \& Wilkins Company, Baltimore (1974).

McManus, M., Lerner, H., Barbour, C., and Robbins, D.R. Assessment of borderline psychopathology in hospitalized adolescents. Journal of the American Academy of Child Psychiatry, 23,685 (1984).

Prusoff, B.A., Klerman, G.L., and Paykel, E.S. Concordance between clinical assessments and patients' self-report in depression. Archives of General Psychiatry, 26, 546 (1972).

Raskin, A., Schulterbrandt, J., Reatig, N., and Rice, C.E. Factors of psychopathology in interview, ward behavior, and self-report ratings of hospitalized depressives. Journal of Consulting Psychology, 31, 270 (1967).

Robbins, D.R., Alessi, N.E., Coor, S.C., Poznanski, E.O., and Yanchyshyn, G. W. Journal of the Academy of Child Psychiatry, 21, 251 (1982).

Smouse, P.E., Feinberg, M., Carroll, B.J., Park, M.H., and Rawson, S.G. The Carroll Rating Scale for Depression: II. Factor analysis of the feature profiles. British Journal of Psychiatry, 138, 201 (1981).

Spitzer, R.L., Endicott, J., and Robins, E. Research Diagnostic Criteria: Rationale and reliability. Archives of General Psychiatry, 38, 733 (1978).

Strober, M., Green, J., and Carlson, G. Phenomenology and subtypes of major depressive disorders in adolescence. Journal of Affective Disorders, 3, 281 (1981). 